Gravity-Driven Instability in a Liquid Film Overlying an Inhomogeneous Porous Layer

This article has been downloaded from IOPscience. Please scroll down to see the full text article.

2011 Chinese Phys. Lett. 28024702

(http://iopscience.iop.org/0256-307X/28/2/024702)

View the table of contents for this issue, or go to the journal homepage for more

Download details:

IP Address: 159.226.231.78

The article was downloaded on 23/03/2012 at 03:27

Please note that terms and conditions apply. 


\title{
Gravity-Driven Instability in a Liquid Film Overlying an Inhomogeneous Porous Layer *
}

\author{
ZHAO Si-Cheng(赵思诚) ${ }^{1}$, LIU Qiu-Sheng(刘秋生) ${ }^{1 * *}$, NGUYEN-THI Henri $^{2}$, BILLIA Bernard $^{2}$ \\ ${ }^{1}$ Key Laboratory of Microgravity, Institute of Mechanics, Chinese Academy of Sciences, Beijing 100190 \\ ${ }^{2}$ IM2NP, UMR CNRS 6137, Universitéd'Aix-Marseille III, 13397 Marseille Cedex 20, France
}

(Received 18 November 2010)

\begin{abstract}
A new model consisting of a liquid film overlying a saturated and inhomogeneous porous layer is investigated. We concentrate on effects of inhomogeneity on transition of instability modes. Influences of the averaged porosity and the gradient of porosity distribution on the instability behaviors of a liquid-porous layer system are emphasized. The average permeability of the porous layer is a key factor to determine the penetration of convection in the system.
\end{abstract}

\section{PACS: 47.20.Dr, 47.55.dm, 47.56.+r DOI: $10.1088 / 0256-307 \mathrm{X} / 28 / 2 / 024702$}

The convective motion in a fluid film ${ }^{[1]}$ can be triggered by buoyancy force ${ }^{[2]}$ (Rayleigh convection), or surface tension force ${ }^{[3]}$ (Marangoni convection). They have the respective equivalences for the fluid saturated in porous media. ${ }^{[4-6]}$ Darcy's law ${ }^{[7]}$ is one of the popular models to describe the motion of fluid in the porous media with low porosity. In the high porosity case, the Brinkman model ${ }^{[8]}$ is often used, which is considered as an extension of the Darcy's equation by adding a Laplacian term analogous to that appearing in the Navier-Stokes equation. According to our experience, when the porosity is not extremely high, using the Brinkman model usually brings some problems of calculation, such as longer computation time, more difficult for convergence, etc.

The onset of pure Rayleigh convection in the superposed liquid-porous (homogeneous) layers, sandwiched by two horizontal infinite rigid and thermal conductive wall, heated from the bottom, was first investigated by Chen et al. ${ }^{[9]}$ Darcy's law together with the Beaver-Joseph condition ${ }^{[10]}$ are applied at the liquid-porous interface. They indicated that the neutral instability curve is normally bimodal, which possesses two local minima. The ratio between the depth of the liquid layer and that of the porous layer $h$ is a key parameter of the system, and it has a critical value $h_{c}=0.13$. When $h<0.13$ the instability is the long-wave mode and the convection appears in both liquid and porous layers, and when $h>0.13$ the instability is called the short-wave mode in which the convection is confined in the liquid film. Later, Straughan et $a l^{[11]}$ and Desaive et $a l^{[12]}$ studied the coupled capillary and buoyancy driven instability in the similar system.

The porous layer considered in the previous works mentioned above was in general assumed being homogeneous. However, the naturally formed porous media do not always have a constant porosity. For instance, in the directional solidification of concentrated alloys, a mushy zone consisting of dendrites immersed in the melt always appears. The mushy layer separates the complete melt region and the solid frozen region, and in the theoretical and computational considerations, it is often regarded as a porous layer with variable permeability. ${ }^{[13]}$ Based on our comprehension to the problems, we are hereby going to focus on the buoyancy-driven instability (Rayleigh convection) in a liquid-porous double-layered system, and set the porous layer to be inhomogeneous, i.e., the present work is a combination of our previous ones. ${ }^{[14,15]}$ Consequently, the effect of inhomogeneity on the instability mode transition is analyzed, and the distribution of velocity and the average permeability of porous layer are presented for two different instability modes.

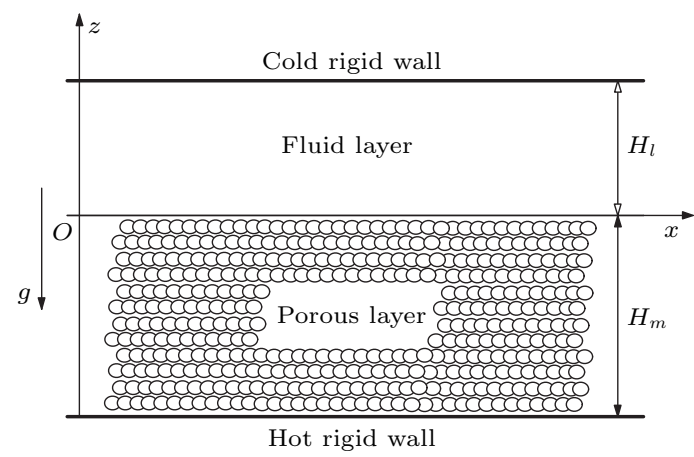

Fig. 1. Geometrical configuration.

The schema of the system is illustrated in Fig. 1. An inhomogeneous porous layer with thickness $H_{m}$ underlies an incompressible liquid layer with thickness $H_{l}$. The two horizontal infinite layers are sandwiched by two parallel, rigid and perfectly heat conductive walls. Cartesian coordinates are introduced with its origin at the fluid-porous interface and the $z$ axis as the normal vector of the boundary walls, which is opposite to the direction of gravitational acceleration.

* Supported by the National Natural Science Foundation of China under Grant Nos 50890182, 11072249 and 10772185 , and the Knowledge Innovation Program of Chinese Academy of Sciences under Grant No KGCX-SW-409.

**Email: liu@imech.ac.cn

(C) 2011 Chinese Physical Society and IOP Publishing Ltd 
The porosity profile is a single valued function of $z$, i.e., it only varies vertically. In the present study, we choose a linear function to describe it. The function is $\phi(z)=\phi_{0}+\phi_{z} \cdot z$, where $z \in[-1,0]$. Here $z$ is in the non-dimensional form shown later, $\phi_{0}$ is the porosity at the fluid-porous interface and is set to be $0.5, \phi_{z}$ is the gradient of porosity distribution.

For an isotropic medium, any physical property has the relation ()$_{m}=()_{l} \phi+()_{s}(1-\phi)$, where $\phi$ represents the local porosity, the subscript $m$ denotes the averaged value, $s$ a property of the solid matrix, and $l$ the property of the interstitial liquid. In order to study the Rayleigh convection, we introduce the Boussinesq approximation

$$
\rho_{l}=\rho_{0}\left[1-\alpha\left(T-T_{0}\right)\right],
$$

where $\alpha$ is the bulk thermal expansion coefficient, $T$ is the temperature, and the subscript 0 represents the value of ambience.

The continuity, momentum and energy equations for liquid layer are

$$
\nabla \cdot \boldsymbol{v}_{l}=0
$$

$$
\begin{gathered}
\rho_{0}\left[\frac{\partial \boldsymbol{v}_{l}}{\partial t}+\left(\boldsymbol{v}_{l} \cdot \nabla\right) \boldsymbol{v}_{l}\right]= \\
-\nabla p_{l}+\mu_{l} \nabla^{2} \boldsymbol{v}_{l}-\rho_{0} g[1 \\
\left.-\alpha\left(T_{l}-T_{0}\right)\right] \boldsymbol{e}_{\boldsymbol{z}} \\
\frac{\partial T_{l}}{\partial t}+\left(\boldsymbol{v}_{l} \cdot \nabla\right) T_{l}=\kappa_{l} \nabla^{2} T_{l} .
\end{gathered}
$$

The equations for the porous layer are

$$
\begin{gathered}
\nabla \cdot \boldsymbol{v}_{m}=0, \\
\frac{\rho_{0}}{\phi} \frac{\partial \boldsymbol{v}_{m}}{\partial t}=-\nabla p_{m}-\rho_{0} g\left[1-\alpha\left(T_{m}-T_{0}\right)\right] \boldsymbol{e}_{z}-\frac{\mu_{l}}{K} \boldsymbol{v}_{m}, \\
(\rho c)_{m} \frac{\partial T_{m}}{\partial t}+\left(\rho_{0} c_{l}\right)\left(\boldsymbol{v}_{m} \cdot \nabla T_{m}\right)=\kappa_{m}\left(\rho_{0} c_{l}\right) \nabla^{2} T_{m} .
\end{gathered}
$$

We introduce perturbations of velocities, pressure and temperature to linearize the equations, and different scales for their non-dimensional form. In the liquid layer, the temperature is scaled by $\Delta T_{l} \nu_{l} / \kappa_{l}$, the length by $H_{l}$, the time by $H_{l}^{2} / \kappa_{l}$, and the velocity by $\nu_{l} / H_{l}$. In the porous layer, they are $\Delta T_{m} \nu_{l} / \kappa_{m 0}$, $H_{m}, H_{m}^{2} / \kappa_{m 0}$, and $\nu_{l} / H_{m}$, where $\kappa_{m 0}=k_{m 0} /\left(\rho_{0} c_{l}\right)=$ $\left[k_{l} \phi_{0}+k_{s}\left(1-\phi_{0}\right)\right] /\left(\rho_{0} c_{l}\right)$. According to the normal mode technique, ${ }^{[16]}$ we seek solutions for the vertical velocity component and temperature in the form:

$$
\left(w_{q}, T_{q}\right)^{T}=\left[W_{q}\left(z_{q}\right), \Theta_{q}\left(z_{q}\right)\right]^{T} \exp \left(\lambda_{q} t_{q}+i a_{q} x_{q}\right) .
$$

The amplitudes $W_{q}$ and $\Theta_{q}$ (where $q=l, m$ ) describe the amplitude of the velocity and temperature, $a_{q}$ are the dimensionless wavenumbers in the $x$-direction, and $\lambda_{q}$ are the complex growth rates of the disturbance. Then the equations (where $\mathrm{D}_{q}=d / d z_{q}$ ) become

$$
\frac{\lambda_{l}}{P r_{l}}\left(\mathrm{D}_{l}^{2}-a_{l}^{2}\right) W_{l}=\left(\mathrm{D}_{l}^{2}-a_{l}^{2}\right)^{2} W_{l}-a_{l}^{2} R a_{l} \Theta_{l}
$$

$$
\lambda_{l} \Theta_{l}=W_{l}+\left(\mathrm{D}_{l}^{2}-a_{l}^{2}\right) \Theta_{l},
$$

$$
\begin{aligned}
& \frac{\lambda_{m}}{\phi P r_{m}}\left(\mathrm{D}_{m}^{2}-a_{m}^{2}\right) W_{m} \\
= & -\frac{1}{\delta_{0}^{2}} f_{1}\left(z_{m}\right)\left[\left(\mathrm{D}_{m}^{2}-a_{m}^{2}\right) W_{m}+\frac{1}{\phi} \frac{d \phi}{d z_{m}} \mathrm{D}_{m} W_{m}\right] \\
& -\frac{R a_{m}}{\delta_{0}^{2}} a_{m}^{2} \Theta_{m}+\frac{1}{\delta_{0}^{2}} f_{1}\left(z_{m}\right) f_{2}\left(z_{m}\right) \mathrm{D}_{m} W_{m} \\
& \lambda_{m} G_{m 0} f_{3}\left(z_{m}\right) \Theta_{m} \\
= & Y \cdot f_{4}^{-1}\left(z_{m}\right) W_{m}+f_{4}\left(z_{m}\right)\left(\mathrm{D}_{m}^{2}-a_{m}^{2}\right) \Theta_{m} \\
& +f_{5}\left(z_{m}\right) \mathrm{D}_{m} \Theta_{m}
\end{aligned}
$$

The dimensionless parameters are

$$
\begin{gathered}
P r_{l}=\frac{\nu_{l}}{\kappa_{l}}, \quad P r_{m}=\frac{\nu_{l}}{\kappa_{m 0}}, \quad X=\frac{\kappa_{l}}{\kappa_{m 0}}=\frac{k_{0}}{k_{m 0}}, \\
\delta_{0}=\frac{\sqrt{K\left(\phi_{0}\right)}}{H_{m}}, G_{m 0}=\frac{(\rho c)_{m 0}}{\rho_{0} c_{l}}, Y=\frac{H_{m}}{k_{m 0} \int_{-H_{m}}^{0} k_{m}^{-1} d z}, \\
R a_{m}=\frac{\alpha \rho_{0} g \Delta T_{m} H_{m} K\left(\phi_{0}\right)}{\mu_{l} \kappa_{m 0}}, \quad R a_{l}=\frac{\alpha \rho_{0} g \Delta T_{l} H_{l}^{3}}{\mu_{l} \kappa_{l}} .
\end{gathered}
$$

The Prandtl number $\operatorname{Pr}$ and the Rayleigh number $R a$ have their respective definitions in the liquid layer (denoted by the subscript $l$ ) and in the porous layer (by $m) . \quad X$ is the ratio of thermal diffusivity (conductivity), and $\delta_{0}$ is the Darcy number. The functions $f_{1}\left(z_{m}\right)$ to $f_{5}\left(z_{m}\right)$ are defined as

$$
\begin{gathered}
f_{1}\left(z_{m}\right)=\frac{K\left(\phi_{0}\right)}{K\left(\phi\left(z_{m}\right)\right)}, \quad f_{2}\left(z_{m}\right)=\frac{1}{K\left(z_{m}\right)} \frac{d K\left(z_{m}\right)}{d z_{m}}, \\
f_{3}\left(z_{m}\right)=\frac{(\rho c)_{m}}{(\rho c)_{m 0}}, f_{4}\left(z_{m}\right)=\frac{k_{m}}{k_{m 0}}, f_{5}\left(z_{m}\right)=\frac{1}{k_{m 0}} \frac{d k_{m}}{d z_{m}} .
\end{gathered}
$$

Equations (9)-(11) give rise to a tenth-order system with the following associated boundary conditions. At $z_{m}=-1$ :

$$
W_{m}=0, \quad \Theta_{m}=0 .
$$

At $z_{l}=z_{m}=0$ :

$$
\begin{aligned}
& W_{l}=h W_{m}, \quad Y h \Theta_{l}=X^{2} \Theta_{m}, \\
& Y \mathrm{D}_{l} \Theta_{l}=X \mathrm{D}_{m} \Theta_{m}, \\
& \mathrm{D}_{l}^{2} W_{l}-\beta \frac{h}{\delta_{0}} \mathrm{D}_{l} W_{l}+\beta \frac{h^{3}}{\delta_{0}} \mathrm{D}_{m} W_{m}=0, \\
& \frac{\lambda_{l}}{P r_{l}} \mathrm{D}_{l} W_{l}-\lambda_{m} \frac{h^{4}}{\phi_{0} P r_{m}} \mathrm{D}_{m} W_{m} \\
& =\mathrm{D}_{l}\left(\mathrm{D}_{l}^{2}-a_{l}^{2}\right) W_{l}+\frac{h^{4}}{\delta_{0}^{2}} \mathrm{D}_{m} W_{m} .
\end{aligned}
$$

At $z_{l}=1$ :

$$
W_{l}=0, \quad \Theta_{l}=0, \quad \mathrm{D}_{l} W_{l}=0 .
$$

The calculations are carried out using the physical properties of water at temperature $T=297.15 \mathrm{~K}$. Its Prandtl number is 6.34. The solid matrix consists of 
glass beads with a nominal diameter of $3 \mathrm{~mm} .{ }^{[17]}$ The Permeability $K(\phi)$ of such a porous medium is obtained using the Kozeny-Carman relation ${ }^{[18]}$

$$
K(\phi)=\frac{d^{2}}{172.8} \frac{\phi^{3}}{(1-\phi)^{2}},
$$

where $d$ is the diameter of the glass beads. The depth of the whole system is $40 \mathrm{~cm}$, and the depth ratio $h=0.05$.

Table 1. Physical properties of water and glass.

\begin{tabular}{rlrl}
\hline$\rho_{0}$ & $=0.997 \times 10^{3} \mathrm{~kg} / \mathrm{m}^{3}$ & $\rho_{s}=2.5 \times 10^{3} \mathrm{~kg} / \mathrm{m}^{3}$ \\
$c_{l}$ & $=4.16 \times 10^{3} \mathrm{~J} / \mathrm{kg} \cdot \mathrm{K}$ & $c_{s}=0.84 \times 10^{3} \mathrm{~J} / \mathrm{kg} \cdot \mathrm{K}$ \\
$\kappa_{l}$ & $=0.145 \times 10^{-6} \mathrm{~m}^{2} / \mathrm{s}$ & $\kappa_{s}=0.201 \times 10^{-6} \mathrm{~m}^{2} / \mathrm{s}$ \\
$\nu_{l}$ & $=0.919 \times 10^{-6} \mathrm{~m}^{2} / \mathrm{s}$ & & \\
\hline
\end{tabular}

The linearized equations (9)-(12) together with their boundary conditions are discretized using the spectral method (Tau-Chebyshev) ${ }^{[19]}$ and are resolved as the general eigenvalue problem. This method has been compared with the published article ${ }^{[9]}$ and verified to be practical with high computational precision in our previous works. ${ }^{[14,15]}$

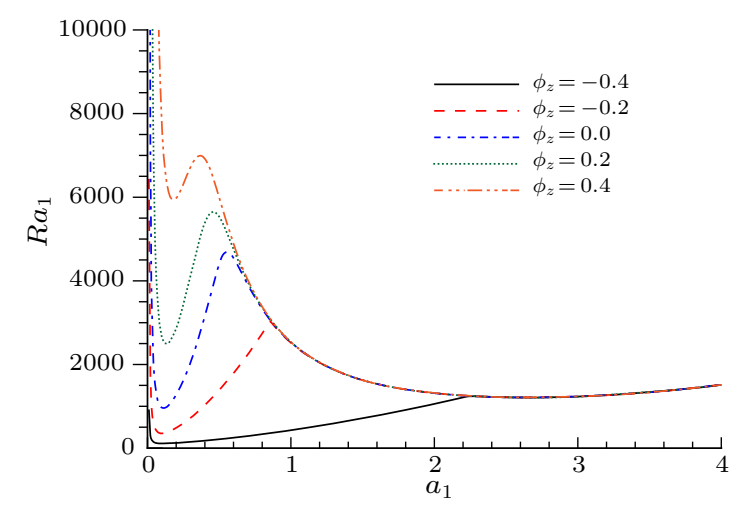

Fig. 2. Marginal curves of instability for different porosity gradient $\phi_{z}$.

Figure 2 illustrates the marginal curves of instability (denoted by $R a_{l}$ and $a_{l}$ ) of the system under the cases of different distribution gradient $\left(\phi_{z}\right)$ in the inhomogeneous porous layer. In a liquid-porous layered system with the homogeneous porous material, the neutral curves are always bimodal, which is first pointed out by Chen et al. ${ }^{[9]}$ Therefore, every neutral curve possesses two branches, and each of them has a local minimum. When the local minimum with small wavenumber is the least value of the whole curve, the convection instability is called the long-wave mode and the convection flow is triggered in both liquid and porous layers. The other case is called the short-wave mode, since the convection appears only in the liquid layer. As shown in Fig. 2, the similar instability modes are also found in the system with an inhomogeneous porous layer, but the transition of instability mode from long-wave to short-wave is rather evident during the increase of $\phi_{z}$ for different porosity distributions. The variation of $\phi_{z}$ only influences the long-wave branch of each neutral curve. The local minimum of the short-wave branch maintains almost the same. This phenomenon can be seen as the effect of permeability of the porous layer. According to the above definition, as a gradient value, $\phi_{z}$ is a key parameter which can operate directly the distribution of porosity, and furthermore, the permeability (from Eq. (13)). The porosity at the liquid-porous interface is 0.5 , and the depth ratio is kept at 0.05 . Hence, the permeability of the porous layer might be the solo factor which has a evident effect on activating the liquid in the porous layer to convect after the system destabilizes.
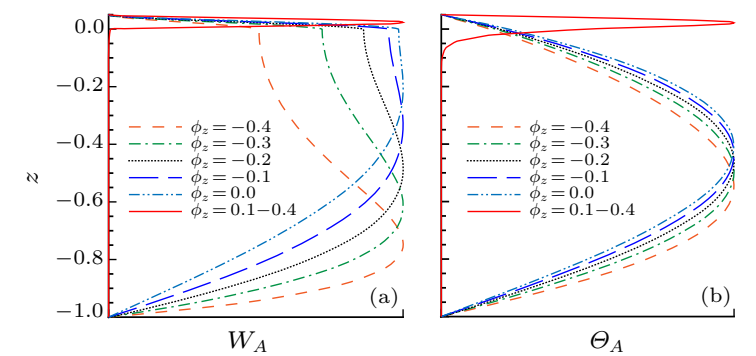

Fig. 3. Amplitudes of velocity (a) and of temperature (b) for different porosity gradient $\phi_{z}$.

The profiles of velocity $W_{q}$ and temperature $\Theta_{q}$ are shown in Fig. 3, respectively. We can see that the porosity gradient $\phi_{z}$ influences significantly the velocity distribution only in the case of long-wave mode instability, in which $\phi_{z}$ is negative. When $\phi_{z}$ turns positive, the convection in the liquid layer is independent of the porosity distribution and the instability of the system corresponds to the short-wave mode. Different from the Marangoni convection in the system with a free surface at the liquid layer, the buoyancydriven instability is triggered in the whole system generally, simultaneously and instantaneously. The convection penetrates most deeply in the porous layer with large porosity. For example, in this system when $\phi_{z}=-0.4$, the largest porosity is at $z_{m}=-1$ and of value 0.9 . Through the amplitudes of temperature, we know that the porosity distribution operates less significantly than it for the velocity in the long-wave mode. Although the temperature perturbation likewise penetrates most deeply in the case of $\phi_{z}=-0.4$, the peak value of its amplitude is not apart from the middle of porous layer very much. It is because water has a relatively stronger viscous effect than its thermal diffusivity $\left(\operatorname{Pr}_{l, m}>1\right)$, and the influence of inhomogeneous permeability is mainly achieved through the viscous resistance imposed on liquid by solid matrix of the porous layer.

In order to study our problem more thoroughly, we introduce the average porosity defined as

$$
\phi_{\text {average }}=\int_{-1}^{0} \phi\left(z_{m}\right) d z_{m} .
$$

Accordingly, a double-layered system mentioned above has a correspondence, where the inhomogeneous porous layer is replaced by a homogeneous one with 
its constant porosity identical to the average value in the former case through the definition of Eq. (14). Hereby, the comparison of neutral curves in the two corresponding systems is illustrated in Fig. 4. We can see that when $\phi_{z}$ is negative, the two local minima of the marginal curves overlap each other, though the forms of the curves are different. However, in each positive $\phi_{z}$ case, only the local minima of the short-wave branch overlap each other. The long-wave branches diverge obviously. These results indicate that, in the case of linearly distributed porosity, its average value, or say, the average permeability, is the key factor to determine the mode of instability.
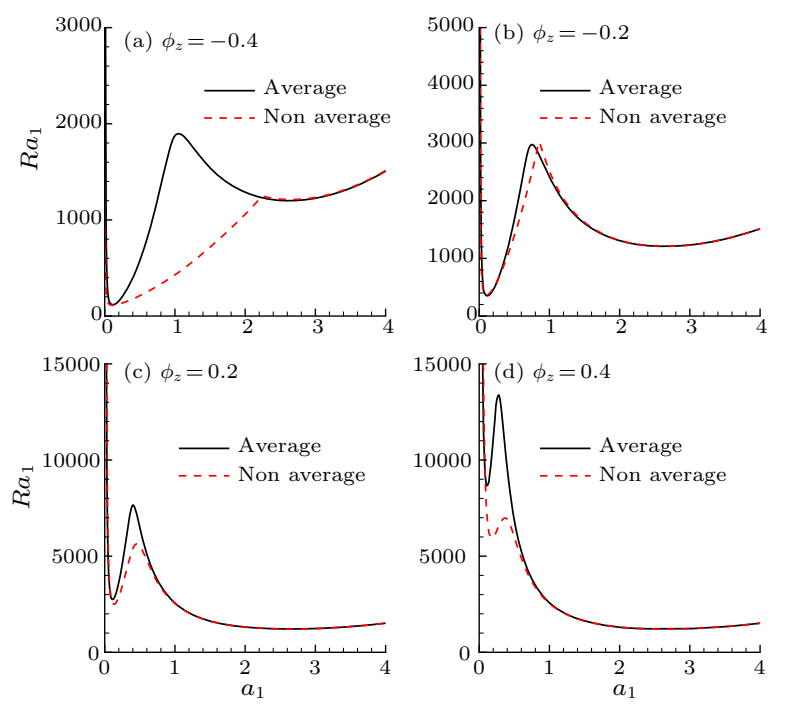

Fig. 4. Comparison of neutral curves between the systems with inhomogeneous porous medium and the cases with the corresponding averaged porosity.

Furthermore, the results in Fig. 4 also indicate the mechanism of the two branches on the neutral curves. The short-wave branch represents the convection of instability only in the liquid layer. Therefore, its characteristics are determined mainly by the properties of the liquid itself. According to the definition of Beavers-Joseph interface condition ${ }^{[10]}$ used, we know that the influence of the porous layer on the liquid layer is realized through the depth ratio $h$, the experiential coefficient $\beta$ and the Darcy number at the interface $\delta_{0}$, fundamentally determined by the porosity there $\phi_{0}$. During our discussion, these three parameters are given and fixed. Hence, the characteristics of the short-wave mode instability can be considered to be independent of the porous layer. This can explain the reason why on the two marginal curves, the local minima, even the shape and trend of the shortwave branch maintain the same always. Contrarily, the long-wave mode instability has a completely different case, because it primarily lies on the porous layer. When and only when the convection can take place in the whole system (corresponding the negative $\phi_{z}$ cases in this study), the local minima of long-wave mode on the two curves can achieve the same value. However, even though at this time, the shapes and trends of the branches are evidently different from each other. The more acutely the porosity varies, i.e., the larger the $\phi_{z}$ is, the more profound the diversity will be. The shape of the long-wave branch just reflects the different porosity distribution.

In summary, in a new model of a double-layered system, the linearly distributed porosity with a variable gradient $\phi_{z}$ in a porous layer can make evidently the transition of instability mode from a short-wave mode to a long-wave mode. The solid matrix of the porous layer can provide the extra viscous dissipation which is strongly dependent on the permeability. The fluid motion in the porous layer with a small porosity is normally more difficult to form than in the case of large porosity. Nevertheless, the effect of solid matrix on the temperature perturbation is much less evident. In addition, we find that the average porosity of the porous layer is the key factor which determines the penetration of convection. The inhomogeneity of the porous layer merely significantly affects the distribution of velocity, and only in the cases that the system destabilizes in the long-wave mode. Accordingly, we can conclude that the average permeability of the porous layer determines the mode of instability, and the inhomogeneity of the porous layer influences the velocity distribution of fluid convection in the porous layer.

\section{References}

[1] Bénard H 1900 Rev. Gen. Sci. Pur. Appl. 111261

[2] Rayleigh L 1916 Phil. Mag. 32(6) 529

[3] Pearson J R A 1958 J. Fluid Mech. 4489

[4] Horton C W and Rogers G T 1945 J. Appl. Phys. 16367

[5] Lapwood E R 1948 Proc. Camb. Philos. Soc. 44508

[6] Hennenberg M, Saghir M Z, Rednikov A and Legros J C 1997 Transport in Porous Media 27327

[7] Nield D A and Bejan A 1998 Convection in Porous Media 2nd edn (New York: Springer)

[8] Brinkman H C 1947 Appl. Sci. Res. A 127

[9] Chen F and Chen C F 1988 J. Heat Transfer 110403

[10] Beavers G S and Joseph D D 1967 J. Fluid Mech. 20197

[11] Straughan B 2001 J. Comput. Phys. 170320

[12] Desaive T and Lebon G 2001 Phys. Rev. E 64066304

[13] Worster M G 1991 J. Fluid Mech. 224335

[14] Zhao S C, Liu R and Liu Q S 2008 Chin. Phys. Lett. 25 620

[15] Zhao S C, Liu Q S, Liu R, Nguyen-Thi H and Billia B 2010 Int. J. Heat Mass Tran. 532951

[16] Colinet P, Legros J C and Velarde M G 2001 Nonlinear Dynamics of Surface-Tension-Driven Instabilities (Berlin: Wiley-VCH)

[17] Chen F and Chen C F 1992 J. Fluid Mech. 23497

[18] Combarnous M A and Bories S A 1975 Adv. Hydrosci. 10 231

[19] Orszag S A 1971 J. Fluid Mech. 50689 